\title{
Seedmarkers: Embeddable Markers for Physical Objects
}

\author{
Christopher Getschmann \\ christopher.getschmann@uni-weimar.de \\ Bauhaus-Universität Weimar \\ Weimar, Germany
}

\author{
Florian Echtler \\ floech@cs.aau.dk \\ Aalborg University \\ Aalborg, Denmark
}

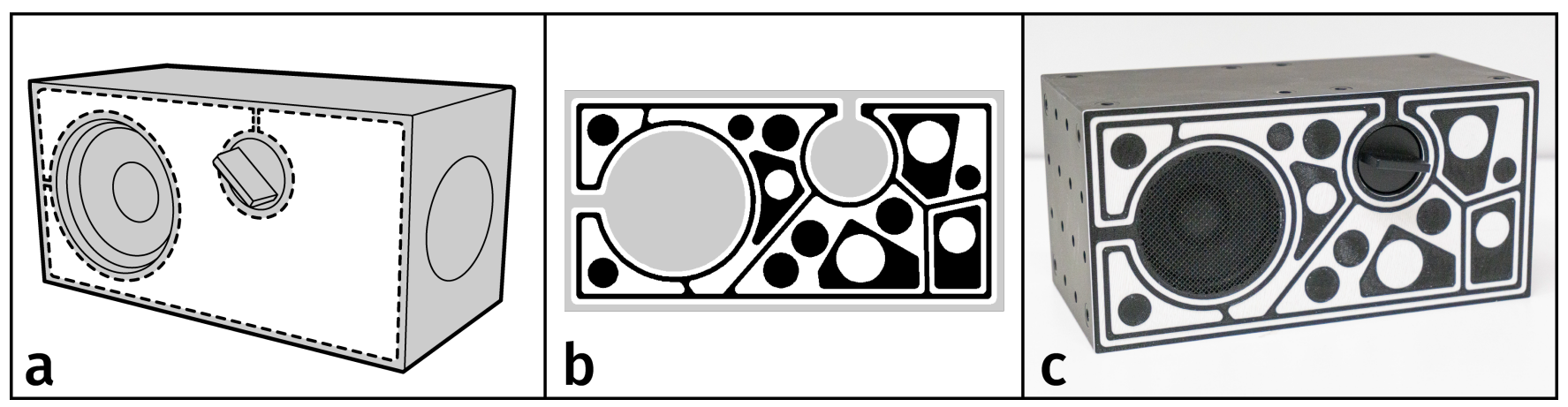

Figure 1: a) Arbitrary shapes on physical objects can be used to allow vision-based interaction with the object itself or its surroundings. b) Given an outline, a marker can be generated and manufactured as a part of the object itself from materials such as wood, acrylic or plastic with $3 \mathrm{D}$ printing or laser cutting

\begin{abstract}
We present Seedmarkers, shape-independent topological markers that can be embedded in physical objects manufactured with common rapid-prototyping techniques. Many markers are optimized for technical performance while visual appearance or the feasibility of permanently merging marker and physical object is not considered. We give an overview of the aesthetic properties of a wide range of existing markers and conducted a short online survey to assess the perception of popular marker designs. Based on our findings we introduce our generation algorithm making use of weighted Voronoi diagrams for topological optimization. With our generator, Seedmarkers can be created from technical drawings during the design process to fill arbitrary shapes on any surface. Given dimensions and manufacturing constraints, different configurations for 3 or 6 degrees of freedom tracking are possible. We propose a set of application examples for shape-independent markers, including 3D printed tangibles, laser cut plates and functional markers on printed circuit boards.
\end{abstract}

\section{CCS CONCEPTS}

- Human-centered computing $\rightarrow$ Ubiquitous and mobile devices;

\footnotetext{
Permission to make digital or hard copies of part or all of this work for personal or classroom use is granted without fee provided that copies are not made or distributed for profit or commercial advantage and that copies bear this notice and the full citation on the first page. Copyrights for third-party components of this work must be honored

For all other uses, contact the owner/author(s).

TEI '21, February 14-17, 2021, Salzburg, Austria

(c) 2021 Copyright held by the owner/author(s).

ACM ISBN 978-1-4503-8213-7/21/02.

https://doi.org/10.1145/3430524.3440645
}

\section{KEYWORDS}

topological markers, rapid prototyping, 3D printing, pose estimation, information embedding

\section{ACM Reference Format:}

Christopher Getschmann and Florian Echtler. 2021. Seedmarkers: Embeddable Markers for Physical Objects. In Fifteenth International Conference on Tangible, Embedded, and Embodied Interaction (TEI '21), February 1417, 2021, Salzburg, Austria. ACM, New York, NY, USA, 11 pages. https: //doi.org/10.1145/3430524.3440645

\section{INTRODUCTION}

When interacting with tangible objects, camera-based detection of their position and their orientation/pose allows rich interfaces and input/output methods. To allow identification and tracking of distinct objects, known natural or artificial visual features of these objects are required.

For this computer vision markers are widely used. Mostly this is done by printing a marker of a fixed size and shape on paper and gluing the paper to a surface of the object. Common 2D codes such as QR-codes, AR-markers or computer vision fiducials are highly visible by design and use a fixed or known geometry to facilitate detection. While several approaches exist to tag and identify physical objects in an invisible or unobtrusive way, these methods are close-range and most do not allow to estimate the full 6 degrees of freedom pose.

We envision a future where devices and household goods are not mass-produced perfect copies but are unique and one-of-a-kind customized objects, tailored to the needs and preferences of their owners. Individual manufacturing of physical goods allows the creation of artificial features, embedded in objects at no further cost. This is already true for prototypes used in research scenarios. While 
most markers and fiducials are optimized towards detection speed [36], robustness [42] or quality of pose-estimation [4, 5], aesthetic considerations are mostly neglected. Any marker system supporting tangible objects in a ubiquitous computing environment or smart home would need to take user acceptance into consideration. Adding known visual features to objects which are either unobtrusive or visually pleasing could allow camera-based interaction with tangibles in an easier, more robust way.

We propose Seedmarkers, a family of generative shape-independent visual codes that are suitable for direct embedding during manufacturing of prototypes and small-size production batches with 3D printing or laser cutting and aim to satisfy basic aesthetic needs (see fig.1).

Existing markers and fiducials can be subdivided into markers which allowing estimation of 6 degrees of freedom (DoF), providing full pose recovery and markers only allowing estimation of 3 or 2 DoF. The proposed Seedmarkers are flexible in shape and complexity and allow multiple modes of operation. Due to supporting arbitrary marker shapes, small and less complex Seedmarkers allow tracking with 3 DoF, suitable for tangible objects on tables or walls. In this configuration, markers can be generated which are compatible with ReacTIVision, allowing seamless integration of our shape-independent markers in many existing ReacTIVision/TUIObased systems. When full 6 DoF pose estimation is required, sideloading of each marker's geometry information is necessary and requires larger markers and thus larger surfaces to support complex structures providing identifiable point correspondences. To allow arbitrary shapes, depending on the geometry of object which needs to be identified and its graspable elements such as knobs, buttons or handles, flexible generation of markers during the design process with CAD-software is necessary.

We contribute:

- a design for complex shape-independent markers based solely on topological structure and the necessary generator to create files for digital manufacturing

- An example implementation of a detector for up to 6 DoF pose estimation

- A technical evaluation of the concept, discussing generator performance and pose estimation error

\section{RELATED WORK}

In general markers are symbols with a known geometry that contain features that are fast and robust to detect based on their shape and contrast or color. Nearly all markers use high-contrast black and white regions, usually produced by Inkjet or laser printing on paper. In wide use are round fiducials and square matrix markers.

\section{Matrix Markers}

Matrix-based markers such as ARToolkitPlus [46], AprilTags [36] and ArUco [41] use a square black border containing a matrix of white and black bits. Visually they closely resemble 2D-Barcodes like DataMatrix or the widely known QR-Code. The pose is estimated by finding the projective-distorted square corners of the marker border and derive orientation information by decoding the
ID data bits in the matrix. The size of the square needs to be known for 6 DoF pose estimation.

\section{Point Markers}

RuneTag [5] and Pi-Tag [6] encode information in a sequence of circles. RuneTag conducts pose estimation by fitting and refining an ellipse, resulting in more precise point correspondences compared to the four corners of a square. Random Dot Markers $[44,45]$ are randomly generated point sets that contain no coded information or geometric relation. The circles comprising the point-based markers are less obtrusive and small, at the cost of lower performance for detecting small or far-away markers.

\section{Topological Markers}

A different approach to detection is encoding information in the topological structure, the hierarchical sequence of black and white regions. The topological structure does not depend on geometry, so any arbitrary shape can be used. The d-touch [10] system and one of the three approaches presented by Nishino [33] allow hand-drawn markers, manually customizable for the given environment and task. ReacTIVision amoeba markers [2] feature a default collection of markers with fixed shapes generated by an evolutionary algorithm. While other shapes are possible, the pre-generated set of markers is optimized for detection robustness and small size. ReacTIVisions markers allow 3 DoF pose estimation by deriving an orientation vector from the placement of white and black leaves. There exist several other approaches to improve topological markers by adding orientation vectors or error-correction codes such as two of the Nishino markers [32, 33] and Yamaarashi [21], creating hybrid markers that rely on topological as well as positional or angle information, requiring a fixed marker shape.

\section{Shape-independent Markers}

While d-touch, ReacTIVision, and the markers by Nishino [33] are shape-independent markers, only ReacTIVision bundles a set of default geometries, the amoeba markers. To create geometries for new graphs, there exist tools such as the FidGen applet ${ }^{1}$. For other solely topology-based markers drawing or creating the symbols itself is a manual task. As an extension to AprilTags [26], the uramaki tag layout has been proposed that allows to manually define ignore regions in matrix blocks. These ignore regions allow to influence the outer boundary of the marker to an extent without intentionally destroying bits recovered by the error-correction. Random Dot Markers are by default shape independent as well.

\section{Aesthetic Markers}

There are a few specialized approaches to create markers that adhere to common aesthetic principles or support customization to allow for a visually appealing integration into images or visual environments. ARTTag [20] integrates circles into specially crafted artwork. Tenmoku et al. [43] proposed fiducials that follow geometric outlines of furniture such as cabinets and shelves to blend in. The point-based Random Dot Markers are unobtrusive in their appearance by design and can be embedded into regular imagery such as maps [48]. Farkas et al. [13] created colored marker elements

\footnotetext{
${ }^{1}$ https://code.google.com/archive/p/fidgen/
} 


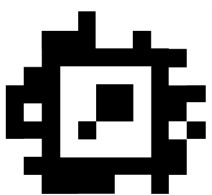

Apriltag [36]

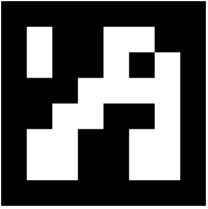

ArUco $[16,41]$

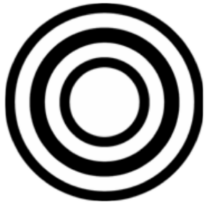

CC-Tag [7]

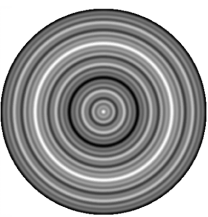

FourierTag [42]

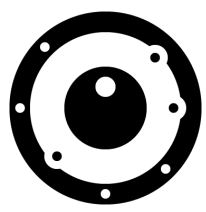

Nishino3 [34]

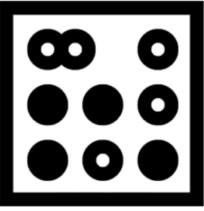

TopoTag [49]*

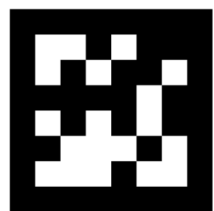

Apriltag [36]

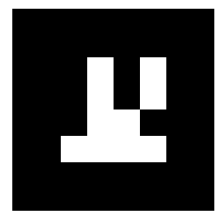

BinARyID [15]

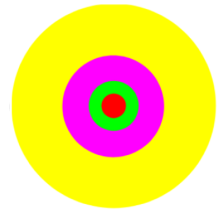

Cho et al. [9]

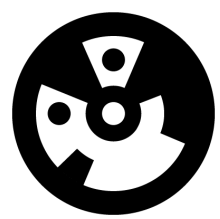

Intersense [31]

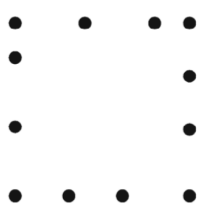

Pi-Tag [6]

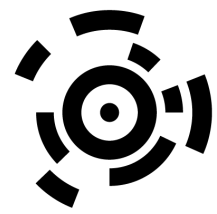

Trip [29]

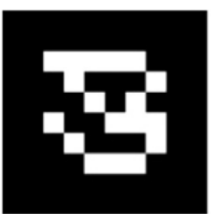

ARtag [14]

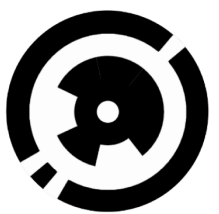

BullsEye [24]

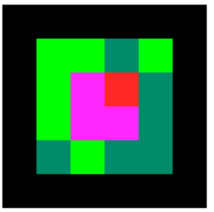

Chromatag [11]

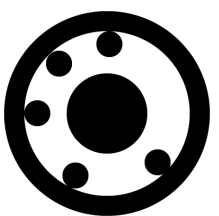

Knyaz et al. [25]

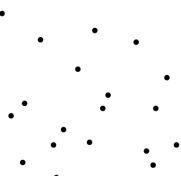

RandomDot $[44,45,48]$

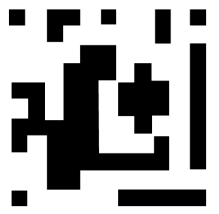

Visual Code [40]

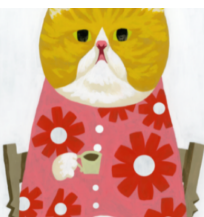

ARTTag [20]

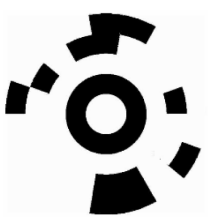

Cantag [39]

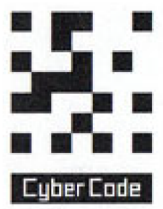

CyberCode [38]

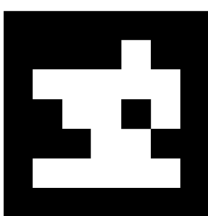

Matrix [37]

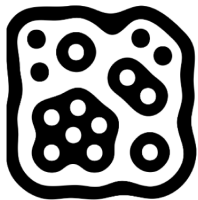

ReacTIVision [2, 3]

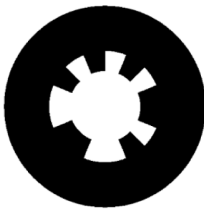

WhyCode [28]

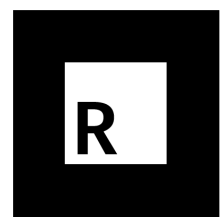

ARtoolkit [22] ${ }^{*}$

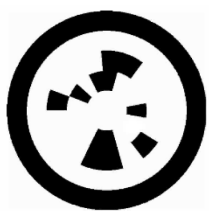

Cantag [39]

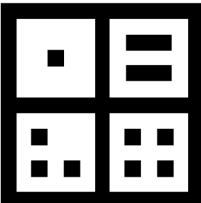

D-Touch [10] *

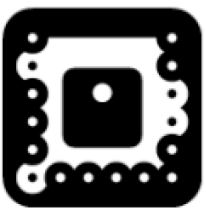

Nishino1 [32]

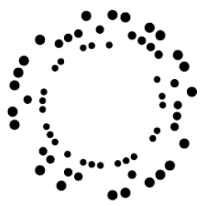

RuneTag [5]

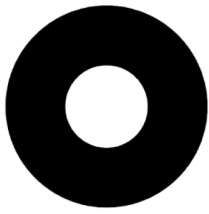

WhyCon [28]

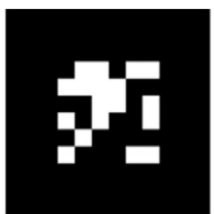

ARtoolkitPlus [46]

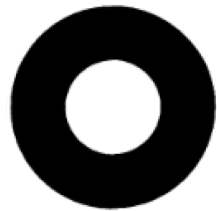

CCC [17]

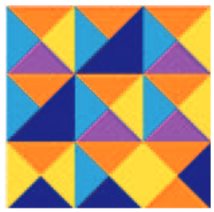

Farkas et al. [13]
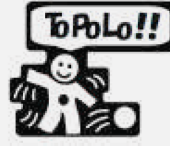

Nishino2 [33] *

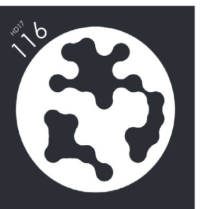

STag [4]

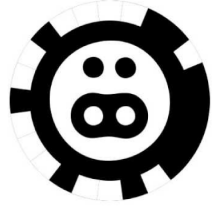

Yamaarashi [21]

Table 1: Visual examples of common Augmented Reality markers and computer vision fiducials. (Example markers are either generated from available code or reprinted with permission) ${ }^{*}$ High visual variety between different instances of the same marker

for indoor robotic navigation that resemble abstract decoration elements. TopoTag [49] are hybrid markers. By using the topology for detection, elements of the marker can be customized but a matrix structure allows error correction and 6-DoF pose estimation. Grinchuk et al. [18] did use neural networks and style transfer techniques to encode and decode data in images for use as markers.
Identification of Printed Objects

For 3D-printed objects, often a suitable way of creating tangibles, both visible as well as invisible techniques have been proposed. From embedding visible bar-codes [30] or QR-Codes [19,23] to invisible methods by using terahertz-scanners [47] for internal structures, utilizing visible pockets and structured light in the material [27] or encoding information in printing parameters and resulting surface imperfections [12]. 
However, these approaches require close-range image data or external hardware such as projectors or specialized cameras and thus are not suitable for larger environments or fast identification and tracking of objects. Only AirCode [27] supports 6 DoF pose estimation.

Manufacturing techniques often used for creating prototypes, such as $3 \mathrm{D}$ printing or laser cutting share common constraints. Especially 3D printing favors rounded corners or circles for small structures. When cut on a laser cutter certain materials such as acrylic are more robust when rectangular shapes are avoided for internal geometries. Depending on process and material, minimum material strengths and geometric constraints influence the size or precision of producing objects with embedded markers. Matrix-based markers require precise 90-degree corners and are thus less suitable for embedding. Any marker that requires color instead of contrast for detection would require an additional process step in manufacturing to add inks or dyes. Point-based markers such as Runetag, Pi-Tag, or the Random Dot Markers are by default simple to manufacture by additive as well as subtractive methods. However, Runetag is limited to a circular shape and Pi-Tag to a square shape, so they can not fully utilize the available space for irregularly shaped objects, but both support cutouts in the center region. Random Dot Markers support arbitrary shapes and cutouts, but detection is complex and knowledge of all dots' relative positions for every single detectable marker is always required.

\section{EXPLORATIVE SURVEY}

To get a better understanding of how different types of markers and their geometric attributes are perceived in everyday living environments, we conducted an online questionnaire. We assume that the willingness of people to accept objects with markers in their personal space is depending either on how aesthetically pleasing or at least how visually unobtrusive they are perceived. Five different markers were selected representatively from a group of popular markers with similar geometric attributes and visual appearance (see fig. 2).

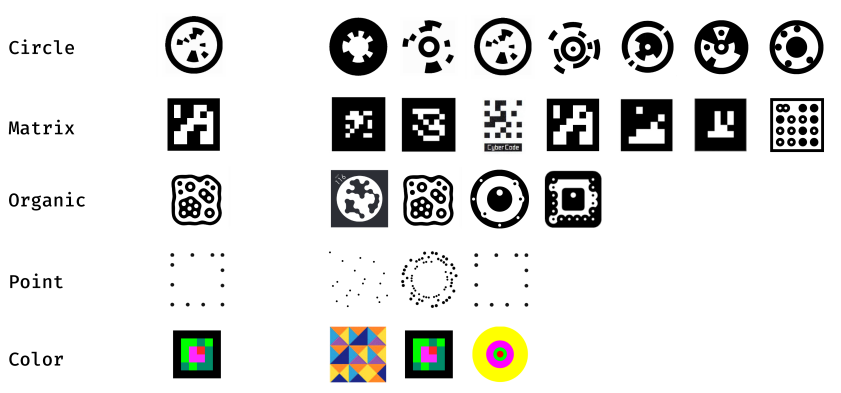

Figure 2: Candidates to represent marker families with similar visual appearance

Each marker was placed on several tangible objects (see fig. 3). As a within-subject study, the images were presented in random order and all participants were asked to rate each marker on a 10point Likert-scale on how „obtrusive“, „aesthetic“ and „acceptable“ a given marker is. In addition to that, participants could give a

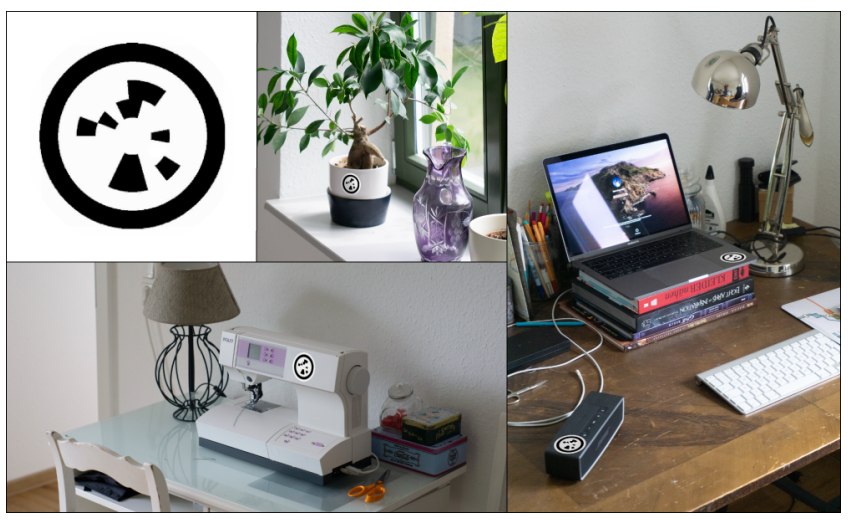

Figure 3: Circle-based marker on tangible and household objects, such as a sewing machine, a speaker, a laptop and a potted plant. Shown as visual examples of a marker in its environment during the survey.

short explanation of their choice. 30 participants completed the questionnaire, 11 female/19 male, ranging from 18 to 35.14 (46 percent) reported not to be familiar with markers at all.

An Omnibus test (Friedman test) was carried out for each variable to see if there were differences in the response based on marker type (non-normally distributed data). There was a statistically significant difference in all three variables: for "obtrusive" $\left(\chi^{2}(4)=50.479\right.$, $p<0.001)$, "aesthetic" $\left(\chi^{2}(4)=17.092, p=0.002\right)$ and "acceptable" $\left(\chi^{2}(4)=13.057, p=0.01\right)$. Based on this each marker was compared with all other markers using the non-parametrical Wilcoxon-signedrank test, resulting in 10 comparisons in total for each variable. See table 2 for significant pairs.

Given Pearsons $r$ and a high $(r>0.5)$ to medium $(r>0.3)$ effect size, all significant comparisons were taken into consideration for our analysis. Additionally, correlation between the three variables was analyzed using the Kendall-Tau-b coefficient. The correlation between aesthetic and acceptable is positive, (Circle: 0.533, Point: 0.450 , Organic: 0.620 , Color: 0.732 , Matrix: $0.527, p<0.001$ each). Thus a marker rated higher in aesthetics is usually rated more acceptable, too. However, the negative correlation between obtrusiveness and acceptance is not significant.

This allows us to focus on median and average values when comparing all five markers. The representatives of the Matrix and Color groups were rated most obtrusive, while Circle and Point markers were most favorable (see fig. 4). In terms of aesthetic Circle and Organic markers were perceived best. Matrix was rated worst. The overall acceptance was highest in the Circle marker. The obtrusiveness-results for Point and Circle are significantly better in comparison to all markers. This is also true for the results of the Point-based marker in terms of acceptance.

The Color marker, in particular, was described by participants as "ugly" and "obtrusive". In addition to that the block-shaped appearance of the Matrix marker is best to be avoided, both in the colorized version and the black and white example. Several participants confused or compared it with QR-Codes, this may influence perception as well. This may be due to the choice of colors to increase contrast 


\begin{tabular}{|c|c|c|c|c|}
\hline & & Obtrusive & Aesthetic & Acceptable \\
\hline Organic & Matrix & $\begin{array}{r}\mathrm{Z}=-2.266 \\
\mathrm{p}=0.023 \\
\mathrm{r}=-0.414\end{array}$ & & \\
\hline Organic & Color & $\begin{array}{r}\mathrm{Z}=-2.245 \\
\mathrm{p}=0.025 \\
\mathrm{r}=-0.410\end{array}$ & & \\
\hline Point & Matrix & $\begin{aligned} \mathrm{Z} & =-4.272 \\
\mathrm{p} & =0.001^{*} \\
\mathrm{r} & =-0.780\end{aligned}$ & & \\
\hline Point & Color & $\begin{aligned} \mathrm{Z} & =-4.488 \\
\mathrm{p} & <0.001^{*} \\
\mathrm{r} & =-0.819\end{aligned}$ & & \\
\hline Point & Organic & $\begin{array}{c}\mathrm{Z}=-3.757 \\
\mathrm{p}<0.001^{*} \\
\mathrm{r}=-0.686\end{array}$ & & \\
\hline Circle & Matrix & $\begin{aligned} \mathrm{Z} & =-3.234 \\
\mathrm{p} & =0.001^{*} \\
\mathrm{r} & =-0.590\end{aligned}$ & $\begin{aligned} \mathrm{Z} & =-3.028 \\
\mathrm{p} & =0.002^{*} \\
\mathrm{r} & =-0.553\end{aligned}$ & $\begin{aligned} \mathrm{Z} & =-2.820 \\
\mathrm{p} & =0.005^{*} \\
\mathrm{r} & =-0.515\end{aligned}$ \\
\hline Circle & Color & $\begin{aligned} \mathrm{Z} & =-2.733 \\
\mathrm{p} & =0.006^{*} \\
\mathrm{r} & =-0.499\end{aligned}$ & & $\begin{array}{r}\mathrm{Z}=-2.231 \\
\mathrm{p}=0.026 \\
\mathrm{r}=-0.407\end{array}$ \\
\hline Circle & Point & $\begin{array}{r}\mathrm{Z}=-2.313 \\
\mathrm{p}=0.021 \\
\mathrm{r}=-0.422\end{array}$ & $\begin{array}{r}\mathrm{Z}=-2.513 \\
\mathrm{p}=0.012 \\
\mathrm{r}=-0.459\end{array}$ & $\begin{array}{r}\mathrm{Z}=-2.574 \\
\mathrm{p}=0.010 \\
\mathrm{r}=-0.470\end{array}$ \\
\hline Circle & Organic & & $\begin{array}{r}\mathrm{Z}=-2.688 \\
\mathrm{p}=0.007 \\
\mathrm{r}=-0.491\end{array}$ & $\begin{array}{r}\mathrm{Z}=-2.241 \\
\mathrm{p}=0.025 \\
\mathrm{r}=-0.409\end{array}$ \\
\hline
\end{tabular}

Table 2: Significant pairwise comparisons resulting from the Friedman Test. * significant after alpha-correction with Bonferroni-Holm

between violet and green. Especially the Circle marker was well regarded and described as a "nice logo" twice. We can conclude that the Point marker is the least obtrusive one while circular shapes may be more obtrusive but rated higher in terms of being aesthetic and acceptable.

\section{SEEDMARKER}

In this section we give a reasoning for the design of Seedmarkers and describe their generation process for arbitrary shapes. Afterward, examples for manufacturing objects with embedded Seedmarkers are presented. The detection of Seedmarkers is discussed only briefly as solely the differences to detecting other topological markers are relevant.

While the perception of aesthetics may differ, it makes sense to focus on reducing obtrusiveness and increasing the aesthetic perception of a marker at the same time. This can be achieved by hiding the solely functional marker behind elements that may be perceived as a purely structural or decorational part of an object. This is easier to achieve if the marker is able to fully utilize the available space on an object. At the same time using larger structures increases detection robustness and suitability for different
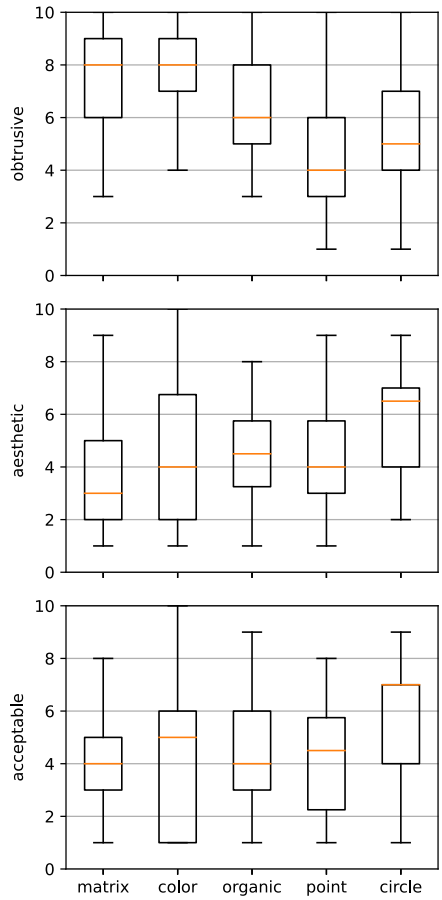

Figure 4: Average, median and standard deviation plot for all three variables (obtrusive, aesthetic, acceptable)

manufacturing techniques. While most marker designs make assumptions about geometric relations such as a matrix or a radial structure, this is not suitable for arbitrary shapes. The topological structure used by topological markers however is independent of the actual shape, only the sequence of encompassing white and black regions encodes information.

This topological structure of a marker can be represented as an undirected acyclic graph, the topology tree. The size of a tree can be described by the maximum number of children of each node (the width) and the maximum distance from root to leaf, its depth. While the order of the subtrees by itself is not unique, $d$-touch and ReacTIVision use a unique representation of this topological tree: when labeling each node by its depth, sorting lexically and doing a depth-first traversal of the tree, the resulting left-heavy-depthsequence is a unique description of a detected topological tree (see fig. 5). See [3] for a detailed explanation.

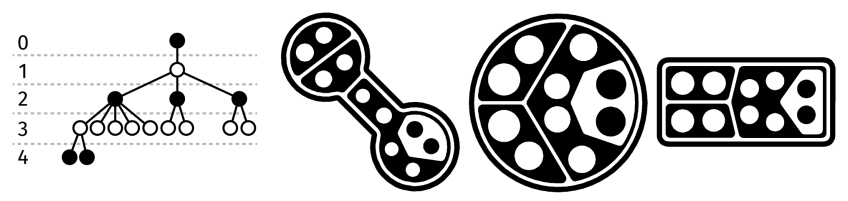

Figure 5: left-heavy-depth-sequence: 0123443333233233, (same topological structure, different shapes) 
To allow a permanent embedding of the marker in the object during fabrication, the specific constraints of common manufacturing methods need to be considered. Laser cutters are limited to connected shapes, no free-floating elements are viable and minimum trace widths depend on the material and its strength. Fused Filament Fabrication 3D printers have strict lower boundaries for precision (usually a multiple of the nozzle size) and rounded geometries result in a more precise extrusion of plastic.

To ensure optimal utilization of space while honoring constraints for manufacturing, the internal geometry of the marker needs to be optimized while keeping the provided topological structure. An optimal geometry would need to satisfy two constraints:

- maximize the minimum size of each topological structure (improves detection from long distances and reduces required precision in the manufacturing process)

- maximize the distance between leaf-elements (reduces error during pose estimation)

When we have a look at nature and how space optimization happens among a large number of different domains, certain visually appealing structures are reoccurring. This is true for the formation of soap-bubbles, morphogenesis in cells or sections in a fruit (see fig.6). These visual elements are identical with geometric attributes of markers that were rated positively in the survey. Seeds in fruits or fur spots are similar to point-patterns. Bubbles tend to arrange themselves in a circular geometry, avoiding sharp edges and favoring organic and smooth transitions.

\subsection{Generation process}

These naturally occurring structures are often modeled with Voronoi diagrams. From a set of points, cells are generated, spanning in each direction half the distance to the next point. Thus size and area of cells is governed by their neighbors' positions.

However, topological trees are not balanced, some subtrees may have considerably more leaves than others. Thus regular Voronoi cells of approximately equal size are not suitable to model structures of unequal complexity.
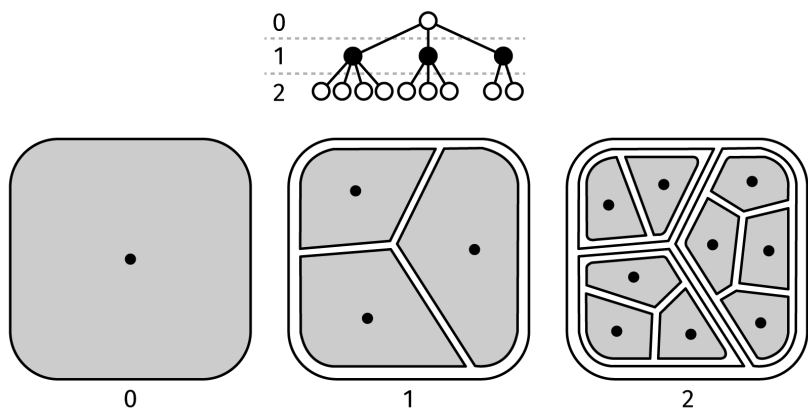

Figure 7: The area is divided by subtree size

By calculating the Voronoi diagram in three dimensions and projecting back to two dimensions, an additional value can be introduced. This distance in the third dimension can be interpreted as a weighting parameter, creating a weighted Voronoi Diagram.
If the distance value is multiplied by itself, this is also known as a Power Diagram [1]. This allows for an efficient subdivision of space as each node of the topological tree is represented by a weighted point corresponding to a Voronoi cell (see fig.7). By assigning each cell a weight similar to the size of the represented subtree they will encompass, an area relative to its weight will be allocated. To improve the circularity of generated cells, Lloyds algorithm is used to iteratively move the Voronoi point to the centroid of its cell (see fig.8).

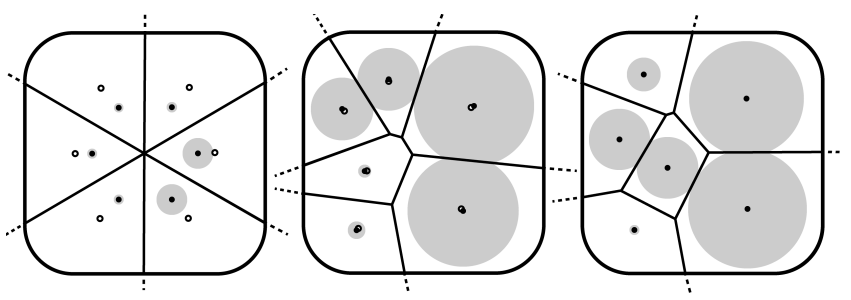

Figure 8: Circular weighted Voronoi diagram using Lloyds algorithm. (Iteration steps: 1, 1000, 3000)

If the error introduced by the relative areas as well as the distances between centroids and cell points falls below a threshold, the node is fixed and its subtrees are optimized. Before fixing a cell and computing cells for subtrees, the polygon representing the cell is shrunk and smoothed in accordance with manufacturing constraints. Fixing subtrees prior to optimizing children nodes increases the convergence speed considerably. This optimization process (see algorithm 1) is repeated recursively for every subtree from root to the leaves. Leaves are represented as the largest circle filling the leaf-region. This has several advantages: in accordance with the user survey the marker has a circular or point-like appearance and it allows the use of ellipse fitting during detection to retrieve precise point correspondences. In addition to that, a circle is the most precise geometry a $3 \mathrm{D}$ printer and a laser cutter can create on small scales.

As a measure of weight for the Voronoi cells the total number of nodes of the corresponding subtree $S$ is used:

$$
w(S)=\alpha *\left|S_{c}\right|+\sum^{c \in S_{c}} w(c)
$$

where $\alpha$ is the depth weighting factor and $c$ the children nodes of the subtree. Using $a>=1$ accounts for deeper subtrees which require more area. Using a more sophisticated heuristic may improve markers for very complex graphs.

Note that this algorithm is sensitive to the initial point placement to achieve an optimal distribution. Instead of random placement, points are aligned on the largest incircle of the boundary polygon. While this step is computationally expensive, it improves the results for markers with very large graphs and objects with complex geometries.

We can conclude that Power diagrams are well suited to represent a topological graph. While their mathematical properties are close to an optimal distribution of space their visual appearance can be easily assumed to be decorational. Another application of power 

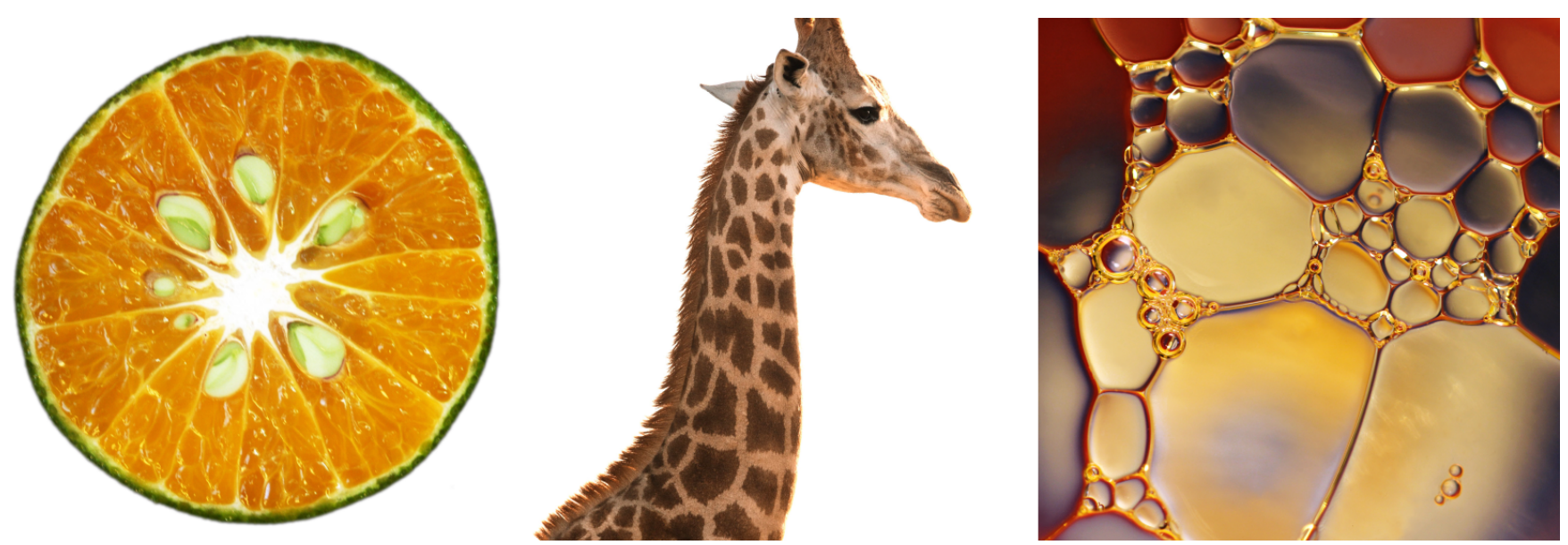

Figure 6: Patterns found in biological or physical processes: slice of an orange, fur patterns of a Giraffe, soap bubbles.

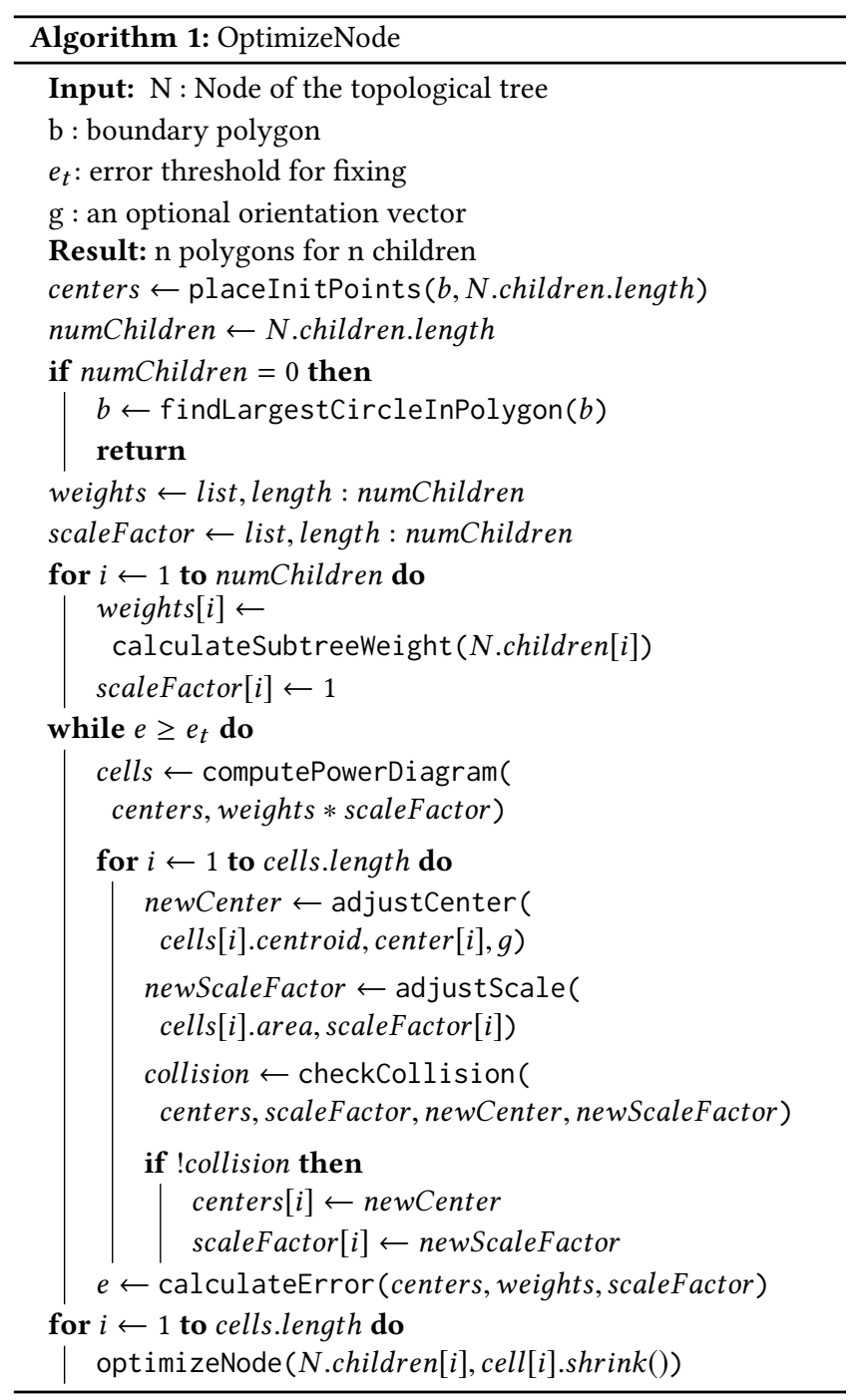

diagrams sharing many attributes with topological optimization are hierarchical treemaps used for data visualization [35], employing identical strategies for optimizing circularity and weighting.

To be able to estimate the 6 DoF pose of a marker, point correspondences and their relative positions need to be acquired. For many markers, the fixed geometry is known and only a physical unit for scaling is required (the border length in matrix-based markers). Since Seedmarkers are shape-independent, both points for point-correspondences and their positions are unknown. The geometry of a generated Seedmarker can be described by the Seedmarker descriptor. This is a string consisting of the name of the marker as a left-heavy-depth-sequence and each leaf's physical coordinates on the object in the units of the technical drawing (usually millimeters). The use of the descriptor for pose estimation is described in section 4.3.

Note that the Seedmarker-generator is deterministic, given the same outline and graph the generated marker will be identical. This is important when the Seedmarker descriptor for a physical object is lost. Given the technical drawings and the topological structure from the physical object itself, the marker and thus the descriptor can be generated again.

\subsection{Manufacturing}

When designing physical objects in CAD-software, the drawing containing the outline of the object can be exported as a DXF-file. The Seedmarker-generator parses this file and outputs the contours of the generated marker in the same format, suitable for re-import.

\section{D printing}

For manufacturing with Fused-Filament-Fabrication 3D printers the object can be designed as usual. Before exporting the object for printing, the markers DXF-file needs to be imported in the CADsoftware and subtracted from the face of the object which will be placed on the build plate of the printer. After printing the first layer with either white or black filament, the printer can pause and let the user switch filaments. This way Seedmarkers can be printed on commodity single-material 3D printers without additional software or pre-processing. Switching filaments after the first layer requires 
only a minimum amount of time and no permanent oversight (see fig.9). If multi-material printers are used, any planar surface of the object is suitable for placement.

\section{Laser cutting}

Engraving the surface before cutting results in clean and precise markers. For some materials, engraving to darken areas may not be suitable. However, during the detection process small structures and noise in the camera image are ignored. This can be exploited to create dark or bright areas by removing most of the material while keeping struts (see fig. 9a).

\section{Other materials}

Very small prototypes may not allow for an enclosure altogether. In this case, it is suitable to place the marker directly on the printed circuit board. This can be done by using silkscreen or exposed copper areas (in this example used for capacitive sensing, see fig.10c).

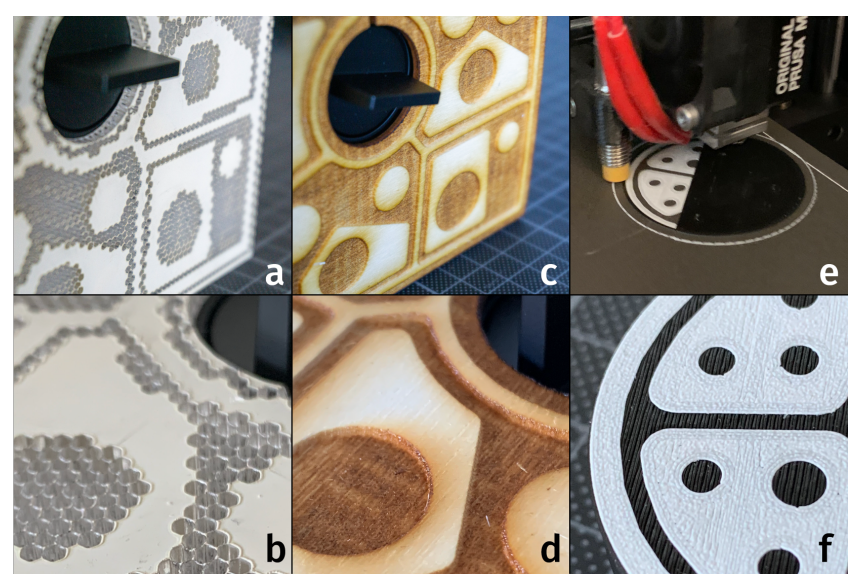

Figure 9: Cutting (a/b) and engraving (c/d) on a laser cutter. Two-color printing separated by layer on a single-color 3D printer (e/f).

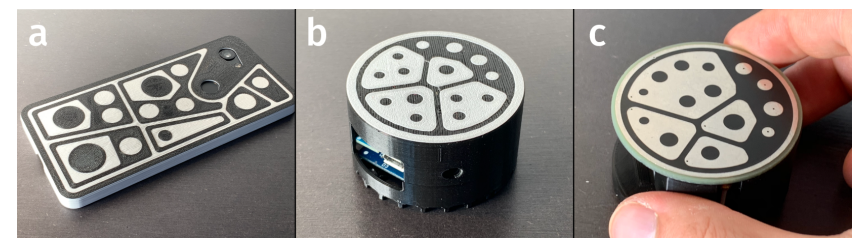

Figure 10: Example applications of Seedmarkers: a) 3D printed smartphone case that allows 2-DoF detection on classic tabletop systems. b) 3D printed tangibles for tablebased interaction compatible with ReacTIVision (3-DoF). c) Marker regions fabricated as exposed copper pours on a PCB, each leaf of the marker doubles as a capacitive touch input.

\subsection{Detection}

The detection of Seedmarkers is conducted in two stages. The first stage is similar to Nishinos [34] approach and ReacTIVisions [2] amoeba marker detection: the topological tree is generated for the full scene and searched for all structures which yield the minimum complexity of a Seedmarker (exactly two nodes without siblings and at least two children). The left-heavy-depth-sequences are generated and recursively compared to known marker names to find marker candidates.

The second stage is optional and only required for pose estimation: Unknown marker names can be used for descriptor sideloading. This can be done by broadcasting the marker name and polling listening devices in the local network to respond with their marker descriptor if their name was detected.

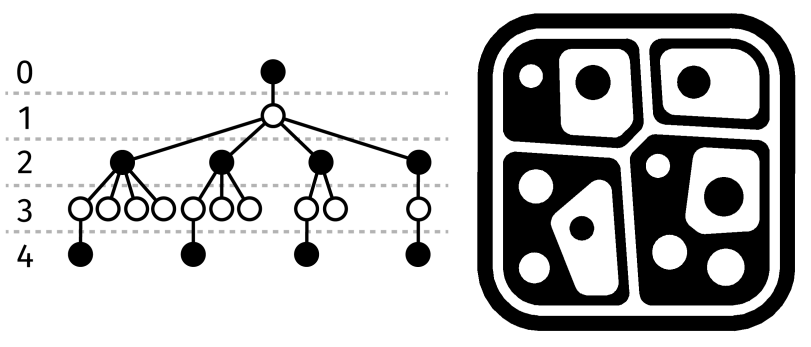

Figure 11: Minimal marker graph with four unique subtrees for $6 \mathrm{DoF}$ pose estimation. Each black leaf (depth 4 ) is unique since the leafs subtree can be uniquely identified based on the number of white leaves at depth 3 .

If the marker descriptor and thus the geometry of all leaf-nodes is known, pose estimation may be possible. To solve the Perspectiven-Point problem, at least 4 point correspondences of marker and image are required. Prerequisite is a topological graph that contains at least four subtrees with a single leaf which can be described by a unique left-heavy-depth-sequence (see fig. 11). This limits 6-DoF pose estimation to a smaller subset of all markers (see table 3). However, given trees with a maximum depth of 4 and a width of up to 4 children per node, the resulting 58905 distinct markers with the ability to estimate 6-DoF pose should be sufficient for many applications.

\begin{tabular}{llrr} 
Depth & Width & Number of markers & 6-DoF \\
\hline \hline 2 & 2 & 3 & - \\
2 & 3 & 4 & - \\
2 & 4 & 5 & - \\
\hline 3 & 2 & 10 & - \\
3 & 3 & 35 & - \\
3 & 4 & 126 & - \\
\hline 4 & 2 & 66 & - \\
4 & 3 & 8436 & - \\
4 & 4 & 11358880 & 58905
\end{tabular}

Table 3: Number of unique topological trees (left-heavydepth-sequences) by maximum width and depth. 


\section{EVALUATION}

Evaluation is divided into three parts: suitable graphs to avoid false positives, generation performance and pose estimation performance.

\subsection{False positives}

When choosing suitable topological graphs to construct markers, randomly or naturally occurring topological structures need to be avoided to reduce false positive detections. Costanza et al. [10] computed for d-touch the total number of false positives on a set of graphs in 10000 random images. The graphs used for matching had exactly a depth of 3 with up to 4 leaves per branch. Since Seedmarkers may be larger in size, the use of wider and deeper graphs is feasible. We repeated this test with a set of all graphs up to depth 6 and width 10. Using 41602 images from the Open Images V4 eval dataset, the topological graphs of the detected false positives are grouped by their maximum depth and width (see fig. 12).

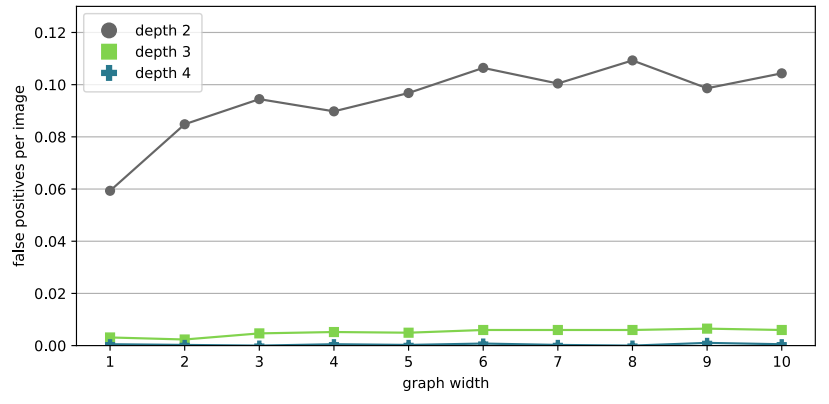

Figure 12: False positive detections per graph family

8.36 percent of all images contained at least one false positive, 3478 of 41602 images in total. 94.4 percent of all false positives are topological structures with a depth of 2 . The number of false positives in relation to graph width does not vary by a large amount. By using graphs with at least one leaf of depth 3 or deeper, we can reduce our chance of a false positive to 0.5 percent per image on the tested dataset.

\subsection{Generator performance}

Given an suitable outline and manufacturing constraints, the generator is able to fill the outline and utilize space well, see fig. 13 for examples. For narrow or concave outlines, the geometrical optimization is sensitive to the distribution of initial placement positions. In the case of colliding and blocking cells, the obstructing cells are frozen to avoid instability which often results in a non-ideal utilization of available space. See fig. 14 for examples of some failure modes.

\subsection{Pose estimation error}

The error of the 6 DoF pose estimation can be evaluated in comparison to a widely used state-of-the-art marker. ArUco is choosen for its common usage in research involving tangibles or augmented reality setups. On artificial images with varying distance and angle,
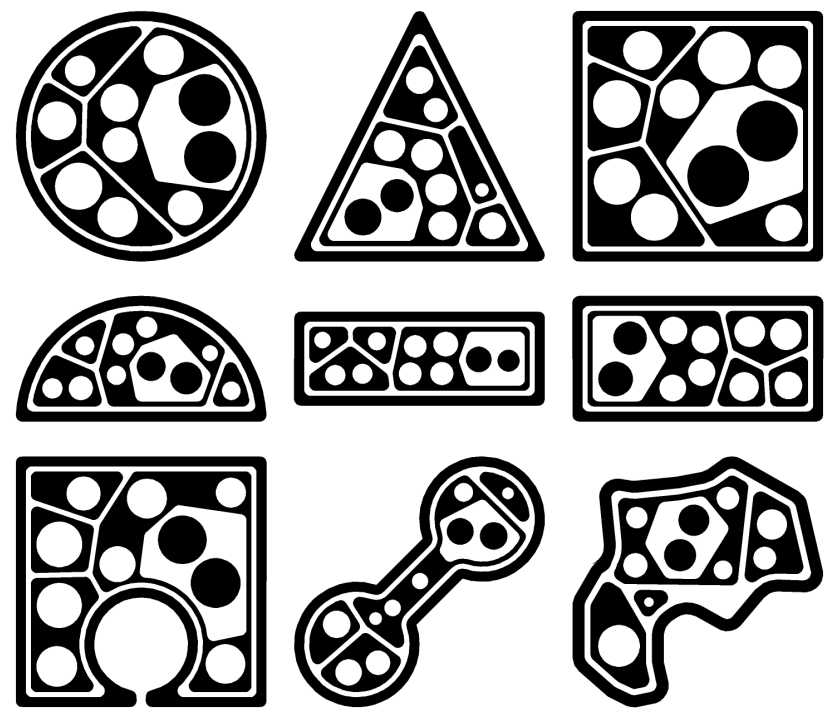

Figure 13: Examples for markers generated with a variety of shapes

a
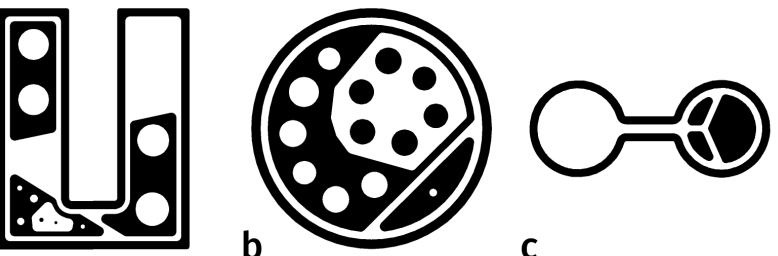

C

Figure 14: Failure cases: a) Concave polygons can yield considerable unused space since Voronoi cells may extend beyond the cavity. b) Large, unbalanced subtrees with two children. c) Narrow passages block cells.

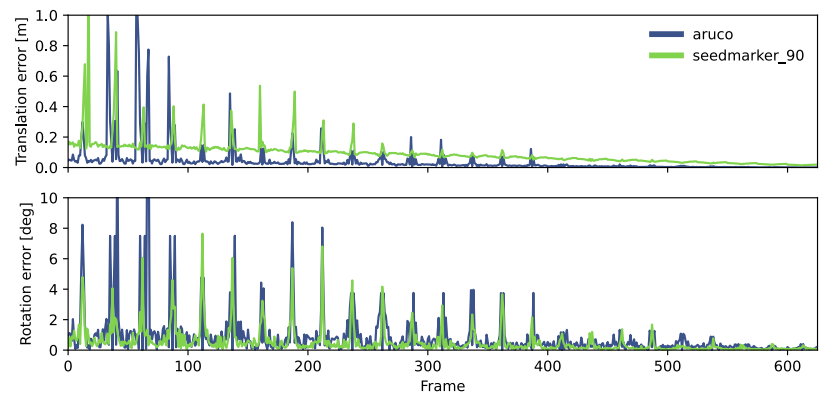

Figure 15: Pose estimation error (translation and rotation) for Seedmarkers and ArUco (familiy 4x4). Both scaled to $90 \mathrm{~mm}$. Distance $2 \mathrm{~m}$ to $0.25 \mathrm{~m}$, angle -45 to +45 degree.

the translation and rotation error is calculated for a Seedmarker and an ArUco marker of equal size (see fig.15). The quality of pose estimation of the Seedmarker is worse than ArUco in regard to average 
translation error as well as rotation error. This results mainly from differences in how point correspondences are detected in the image. ArUco relies for point correspondences on the corners of a square while Seedmarkers rely on the centers of the leaf-circles. Due to the projective distortion by the camera, a square is rendered to a quadrilateral while the circle becomes an ellipse. While the corner points of a quadrilateral still allow precise matching, the center of the ellipse is shifted. By observing multiple coplanar ellipses that are provided by every Seedmarker, this offset can be calculated to refine point positions [8], however, this is not done by the detector yet.

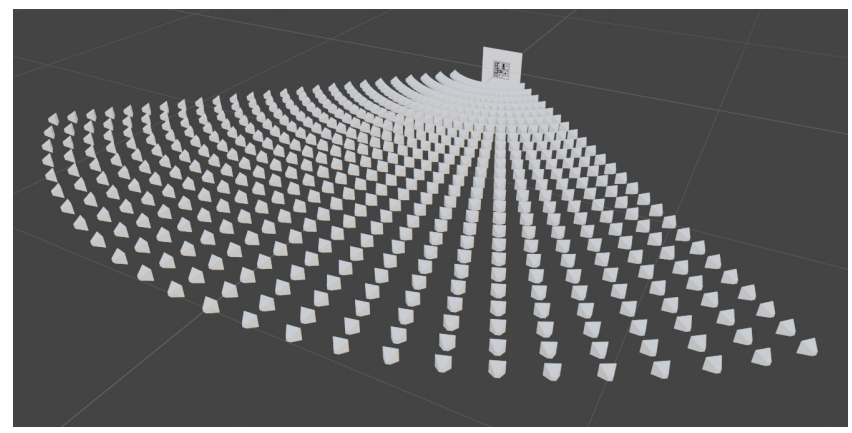

Figure 16: Camera positions to render artificial images for pose estimation

\section{DISCUSSION AND FUTURE WORK}

Measuring aesthetic perception is hard and subject to a number of factors ranging from environment and cultural background to personal preference and many more. However, preferences and rankings of simple geometrical shapes may pave a path towards more aesthetic and acceptable markers for many scenarios. The visual elements we identified as acceptable (circles and circularity, points as well as organic and smooth transitions) are suitable for marker design in general. Using the topological structure of an object for detection and identification gives enough freedom to create markers that adhere to the observed user preferences, but has two major disadvantages. One is the lack of error correction, thus Seedmarkers can not deal with occlusion and are less robust than other marker designs. If elements like knobs or switches are used on the surface of the object, detection is very susceptible to the viewing angle since any protrusion blocking a topological element renders the marker undetectable. Another disadvantage that is inherent to shape-independent markers is the need to obtain geometry information for individual markers to estimate the 6-DoF pose. This requires either physical objects equipped with components able to broadcast their appearance or a managed database of known markers. At the same time the pose estimation requires four unique subtrees in a marker with single leaves since point correspondences are derived from the topological structure. This point registration problem of matching marker leaves to corresponding circles in the image could be solved for an arbitrary number of nodes, allowing pose estimation for less complex graphs and improving the overall precision.
While keeping the manufacturing constraints and the topological structure of the marker intact, there is extensive room for customization for specific scenarios. A co-creation approach using an interactive web tool to let users influence the generation of markers could result in markers even more suitable for various scenarios and would allow us to learn more about aesthetic preferences in marker usage.

\section{CONCLUSION}

We presented Seedmarkers, a novel approach to generate shapeindependent topological markers suitable for embedding in objects during manufacturing with common rapid prototyping methods. With embedded Seedmarkers objects can communicate their appearance for identification and detection with a minimal amount of data. Generation of markers is flexible in terms of required space and shape, detection distance as well as manufacturing technique. The design of our marker is based on power diagrams for topological optimization, resulting in a smooth and organic, bubble-like appearance. These geometrical elements were rated most favorably in a survey comparing five common marker types.

\section{REPRODUCTION NOTE}

The application, source code including documentation, data, and scripts for generating all referenced plots is available publicly: https://github.com/volzotan/Seedmarkers

\section{ACKNOWLEDGMENTS}

This work was funded by the Deutsche Forschungsgemeinschaft (DFG, German Research Foundation) through project EC437/1-1. We would like to thank Alexandre Devert for permission to use his code for efficient power diagram calculation and Marie Bornemann for extensive advice on statistical analysis.

\section{REFERENCES}

[1] Franz Aurenhammer. 1987. Power Diagrams: Properties, Algorithms and Applications. SIAM 7. Comput. 16, 1 (Feb. 1987), 78-96. https://doi.org/10.1137/0216006 Publisher: Society for Industrial and Applied Mathematics.

[2] Ross Bencina and Martin Kaltenbrunner. 2005. The design and evolution of fiducials for the reactivision system. In Proceedings of the Third International Conference on Generative Systems in the Electronic Arts, Vol. 2.

[3] Ross Bencina, Martin Kaltenbrunner, and Sergi Jorda. 2005. Improved Topological Fiducial Tracking in the reacTIVision System. In 2005 IEEE Computer Society Conference on Computer Vision and Pattern Recognition (CVPR'05) - Workshops, Vol. 3. IEEE, San Diego, CA, USA, 99-99. https://doi.org/10.1109/CVPR.2005.475

[4] Burak Benligiray, Cihan Topal, and Cuneyt Akinlar. 2019. STag: A stable fiducial marker system. Image and Vision Computing 89 (Sept. 2019), 158-169. https: //doi.org/10.1016/j.imavis.2019.06.007

[5] Filippo Bergamasco, Andrea Albarelli, Emanuele Rodolà, and Andrea Torsello. 2011. RUNE-Tag: A high accuracy fiducial marker with strong occlusion resilience. In CVPR 2011. 113-120. https://doi.org/10.1109/CVPR.2011.5995544 ISSN: 10636919.

[6] Filippo Bergamasco, Andrea Albarelli, and Andrea Torsello. 2013. Pi-Tag: a fast image-space marker design based on projective invariants. Machine Vision and Applications 24, 6 (Aug. 2013), 1295-1310. https://doi.org/10.1007/s00138-0120469-6

[7] Lilian Calvet, Pierre Gurdjos, and Vincent Charvillat. 2012. Camera tracking using concentric circle markers: Paradigms and algorithms. In 2012 19th IEEE International Conference on Image Processing. 1361-1364. https://doi.org/10.1109/ ICIP.2012.6467121 ISSN: 2381-8549.

[8] Qian Chen, Haiyuan Wu, and Toshikazu Wada. 2004. Camera Calibration with Two Arbitrary Coplanar Circles. In Computer Vision - ECCV 2004 (Lecture Notes in Computer Science), Tomáś Pajdla and Jiří Matas (Eds.). Springer, Berlin, Heidelberg, 521-532. https://doi.org/10.1007/978-3-540-24672-5_41 
[9] Youngkwan Cho, Jongweon Lee, and Ulrich Neumann. 1998. A Multi-ring Color Fiducial System and an Intensity-invariant Detection Method for Scalable FiducialTracking Augmented Reality. In In IWAR. 147-165.

[10] Enrico Costanza and John Robinson. 2003. A Region Adjacency Tree Approach to the Detection and Design of Fiducials. In in Video, Vision and Graphics. 63-69.

[11] Joseph DeGol, Timothy Bretl, and Derek Hoiem. 2017. ChromaTag: A Colored Marker and Fast Detection Algorithm. 1481-1490. https://doi.org/10.1109/ICCV. 2017.164

[12] Mustafa Doga Dogan, Faraz Faruqi, Andrew Day Churchill, Kenneth Friedman, Leon Cheng, Sriram Subramanian, and Stefanie Mueller. 2020. G-ID: Identifying 3D Prints Using Slicing Parameters. In Proceedings of the 2020 CHI Conference on Human Factors in Computing Systems (CHI '20). Association for Computing Machinery, New York, NY, USA, 1-13. https://doi.org/10.1145/3313831.3376202

[13] Zita V. Farkas, Péter Korondi, Dániel Illy, and Lóránt Fodor. 2012. Aesthetic marker design for home robot localization. In IECON 2012 - 38th Annual Conference on IEEE Industrial Electronics Society. 5510-5515. https://doi.org/10.1109/IECON. 2012.6388951 ISSN: 1553-572X

[14] Mark Fiala. 2005. ARTag, a fiducial marker system using digital techniques. In 2005 IEEE Computer Society Conference on Computer Vision and Pattern Recognition (CVPR'05), Vol. 2. 590-596 vol. 2. https://doi.org/10.1109/CVPR.2005.74 ISSN: 1063-6919.

[15] Daniel Flohr and Jan Fischer. 2007. A Lightweight ID-Based Extension for Marker Tracking Systems. In Eurographics Symposium on Virtual Environments, Shor Papers and Posters, Bernd Froehlich, Roland Blach, and Robert van Liere (Eds.) The Eurographics Association. https://doi.org/10.2312/PE/VE2007Short/059-064

[16] Sergio Garrido-Jurado, Rafael Muñoz-Salinas, Francisco Madrid-Cuevas, and Rafael Medina-Carnicer. 2015. Generation of fiducial marker dictionaries using Mixed Integer Linear Programming. Pattern Recognition 51 (10 2015). https //doi.org/10.1016/j.patcog.2015.09.023

[17] Lance B. Gatrell, William A. Hoff, and Cheryl W. Sklair. 1992. Robust image features: concentric contrasting circles and their image extraction, William E. Stoney (Ed.). Boston, MA, 235-244. https://doi.org/10.1117/12.56761

[18] Oleg Grinchuk, Vadim Lebedev, and Victor Lempitsky. 2016. Learnable visual markers. In Proceedings of the 30th International Conference on Neural Information Processing Systems (NIPS'16). Curran Associates Inc., Red Hook, NY, USA, 41504158.

[19] Sinan Gültekin, Ahmet Ural, and Ulas Yaman. 2019. Embedding QR Codes on the Interior Surfaces of FFF Fabricated Parts. Procedia Manufacturing 39 (Jan. 2019), 519-525. https://doi.org/10.1016/j.promfg.2020.01.411

[20] Shinichi Higashino, Sakiko Nishi, and Ryuuki Sakamoto. 2016. ARTTag: aesthetic fiducial markers based on circle pairs. In ACM SIGGRAPH 2016 Posters (SIGGRAPH '16). Association for Computing Machinery, New York, NY, USA, 1-2. https: //doi.org/10.1145/2945078.2945116

[21] Martin Kaltenbrunner. 2017. An abstraction framework for tangible interactive surfaces. Ph.D. Dissertation. Bauhaus-Universität Weimar. https://doi.org/10. 25643/bauhaus-universitaet.3717

[22] Hirokazu Kato and Mark Billinghurst. 1999. Marker tracking and HMD calibration for a video-based augmented reality conferencing system. In Proceedings 2nd IEEE and ACM International Workshop on Augmented Reality (IWAR'99). 85-94. https://doi.org/10.1109/IWAR.1999.803809

[23] Ryosuke Kikuchi, Sora Yoshikawa, Pradeep Kumar Jayaraman, Jianmin Zheng, and Takashi Maekawa. 2018. Embedding QR codes onto B-spline surfaces for 3D printing. Computer-Aided Design 102 (Sept. 2018), 215-223. https://doi.org/10. 1016/j.cad.2018.04.025

[24] Clemens Nylandsted Klokmose, Janus Bager Kristensen, Rolf Bagge, and Kim Halskov. 2014. BullsEye: High-Precision Fiducial Tracking for Table-Based Tangible Interaction. In Proceedings of the Ninth ACM International Conference on Interactive Tabletops and Surfaces (Dresden, Germany) (ITS '14). Association for Computing Machinery, New York, NY, USA, 269-278. https: //doi.org/10.1145/2669485.2669503

[25] Vladimir A. Knyaz and Alexandr V. Sibiryakov. 1998. The development of new coded targets for automated point identification and non-contact 3D-surface measurements. In 3D Surface Measurements, International Archives of Photogrammetry and Remote Sensing, Vol. XXXII, part 5. 80-85.

[26] Maximilian Krogius, Acshi Haggenmiller, and Edwin Olson. 2019. Flexible Layouts for Fiducial Tags. In 2019 IEEE/RS7 International Conference on Intelligent Robots and Systems (IROS). 1898-1903. https://doi.org/10.1109/IROS40897.2019. 8967787

[27] Dingzeyu Li, Avinash S. Nair, Shree K. Nayar, and Changxi Zheng. 2017. AirCode: Unobtrusive Physical Tags for Digital Fabrication. In Proceedings of the 30th Annual ACM Symposium on User Interface Software and Technology. ACM, Québec City OC Canada, 449-460. https://doi.org/10.1145/3126594.3126635

[28] Peter Lightbody, Tomáš Krajník, and Marc Hanheide. 2017. A versatile highperformance visual fiducial marker detection system with scalable identity encoding. In Proceedings of the Symposium on Applied Computing (SAC '17) Association for Computing Machinery, New York, NY, USA, 276-282. https //doi.org/10.1145/3019612.3019709
[29] Diego Lopez de Ipina, Paulo R. S. Mendonça, Andy Hopper, and Andy Hopper. 2002. TRIP: A Low-Cost Vision-Based Location System for Ubiquitous Computing. Personal and Ubiquitous Computing 6, 3 (May 2002), 206-219. https://doi.org/10. 1007/s007790200020

[30] Henrique Teles Maia, Dingzeyu Li, Yuan Yang, and Changxi Zheng. 2019. LayerCode: optical barcodes for 3D printed shapes. ACM Transactions on Graphics 38, 4 (July 2019), 112:1-112:14. https://doi.org/10.1145/3306346.3322960

[31] Leonid Naimark and Eric Foxlin. 2002. Circular data matrix fiducial system and robust image processing for a wearable vision-inertial self-tracker. In Proceedings. International Symposium on Mixed and Augmented Reality. 27-36. https://doi. org/10.1109/ISMAR.2002.1115065

[32] Hiroki Nishino. 2010. A 6 DoF fiducial tracking method based on topological region adjacency and angle information for tangible interaction. In Proceedings of the fourth international conference on Tangible, embedded, and embodied interaction (TEI '10). Association for Computing Machinery, New York, NY, USA, 253-256. https://doi.org/10.1145/1709886.1709937

[33] Hiroki Nishino. 2010. A shape-free, designable 6-DoF marker tracking method. In ACM SIGGRAPH 2010 Posters (SIGGRAPH '10). Association for Computing Machinery, New York, NY, USA, 1. https://doi.org/10.1145/1836845.1836993

[34] Hiroki Nishino. 2010. Topolo Surface: A 2D Fiducial Tracking System Based on Topological Region Adjacency and Angle Information. fournal of Information Processing 18 (2010), 16-25. https://doi.org/10.2197/ipsjjip.18.16

[35] Arlind Nocaj and Ulrik Brandes. 2012. Computing Voronoi Treemaps: Faster, Simpler, and Resolution-independent. Computer Graphics Forum 31, 3pt1 (June 2012), 855-864. https://doi.org/10.1111/j.1467-8659.2012.03078.x

[36] Edwin Olson. 2011. AprilTag: A robust and flexible visual fiducial system. In Proceedings of the IEEE International Conference on Robotics and Automation (ICRA). IEEE, 3400-3407. https://doi.org/10.1109/ICRA.2011.5979561

[37] Jun Rekimoto. 1998. Matrix: a realtime object identification and registration method for augmented reality. In Proceedings. 3rd Asia Pacific Computer Human Interaction (Cat. No.98EX110). 63-68. https://doi.org/10.1109/APCHI.1998.704151

[38] Jun Rekimoto and Yuji Ayatsuka. 2000. CyberCode: designing augmented reality environments with visual tags. In Proceedings of DARE 2000 on Designing augmented reality environments (DARE ' 00 ). Association for Computing Machinery, New York, NY, USA, 1-10. https://doi.org/10.1145/354666.354667

[39] Andrew Rice, Alastair R. Beresford, and Robert K. Harle. 2006. Cantag: an open source software toolkit for designing and deploying marker-based vision systems. In Fourth Annual IEEE International Conference on Pervasive Computing and Communications (PERCOM'06). 10 pp.-21. https://doi.org/10.1109/PERCOM. 2006.13

[40] Michael Rohs. 2005. Real-World Interaction with Camera Phones. In Ubiquitous Computing Systems (Lecture Notes in Computer Science), Hitomi Murakami, Hideyuki Nakashima, Hideyuki Tokuda, and Michiaki Yasumura (Eds.). Springer, Berlin, Heidelberg, 74-89. https://doi.org/10.1007/11526858 7

[41] Francisco Romero-Ramirez, Rafael Muñoz-Salinas, and Rafael Medina-Carnicer. 2018. Speeded Up Detection of Squared Fiducial Markers. Image and Vision Computing 76 (06 2018). https://doi.org/10.1016/j.imavis.2018.05.004

[42] Junaed Sattar, Eric Bourque, Philippe Giguere, and Gregory Dudek. 2007. Fourier tags: Smoothly degradable fiducial markers for use in human-robot interaction. In Fourth Canadian Conference on Computer and Robot Vision (CRV '07). 165-174. https://doi.org/10.1109/CRV.2007.34

[43] Ryuhei Tenmoku, Yusuke Yoshida, Fumihisa Shibata, Asako Kimura, and Hideyuki Tamura. 2007. Visually Elegant and Robust Semi-Fiducials for Geometric Registration in Mixed Reality. In 2007 6th IEEE and ACM International Symposium on Mixed and Augmented Reality. 261-262. https://doi.org/10.1109/ ISMAR.2007.4538857

[44] Hideaki Uchiyama and Yuji Oyamada. 2018. Transparent Random Dot Markers. In 2018 24th International Conference on Pattern Recognition (ICPR). 254-259. https://doi.org/10.1109/ICPR.2018.8545845 ISSN: 1051-4651.

[45] Hideaki Uchiyama and Hideo Saito. 2011. Random dot markers. In VR 2011 IEEE Virtual Reality 2011, Proceedings. 271-272. https://doi.org/10.1109/VR.2011. 5759503

[46] Daniel Wagner and Dieter Schmalstieg. 2007. Artoolkitplus for pose tracking on mobile devices. In In Computer Vision Winter Workshop 2007 (CVWW'07). Computer Vision Winter Workshop 2007 (CVWW'07).

[47] Karl D. D. Willis and Andrew D. Wilson. 2013. InfraStructs: fabricating information inside physical objects for imaging in the terahertz region. ACM Transactions on Graphics 32, 4 (July 2013), 138:1-138:10. https://doi.org/10.1145/ 2461912.2461936

[48] Liming Yang, Jean-Marie Normand, and Guillaume Moreau. 2014. Robust random dot markers: towards augmented unprepared maps with pure geographic features. In Proceedings of the 20th ACM Symposium on Virtual Reality Software and Technology (VRST '14). Association for Computing Machinery, New York, NY, USA, 45-54. https://doi.org/10.1145/2671015.2671022

[49] Guoxing Yu, Yongtao Hu, and Jingwen Dai. 2020. TopoTag: A Robust and Scalable Topological Fiducial Marker System. IEEE Transactions on Visualization and Computer Graphics (2020). https://doi.org/10.1109/tvcg.2020.2988466 